\title{
Raman fiber laser with highly non-linear fiber
}

\begin{abstract}
This paper demonstrates the power performance of an asymmetrical distributed feedback Raman fiber laser (DF-RFL) employing $2 \mathrm{~km}$ of highly nonlinear fiber (HNLF). The forward-pumped architecture exhibited better threshold condition and higher output power alongside a broader spectral profile compared to a DF-RFL utilizing a standard transmission fiber. This experimental layout could offer better insight on flatter broadband gain compared to conventional DF-RFL.
\end{abstract}

Keyword: Distributed feedback Raman fiber laser; Highly nonlinear fiber (HNLF); Random laser; Rayleigh scattering; Stimulated Raman scattering (SRS) 